\title{
Aspectos psicosociales asociados en el proceso de inclusión educativa durante la infancia intermedia
}

\author{
Psychosocial Aspects Associated with the Educational Inclusion Process During Middlle Childhood \\ Reyna de los Ángeles Campa Álvarez Carlos René Contreras Cázarez²
}

Para citar este artículo: Campa, R. Á., Contreras, C. R. (2018). Aspectos psicosociales asociados en el proceso de inclusión educativa durante la infancia intermedia. Infancias Imágenes, 17(1), 9-24.

\section{Resumen}

El objetivo principal del presente trabajo es analizar los aspectos psicosociales (actitudes, cultura de la diversidad y práctica docente) para determinar su relación en el proceso de inclusión educativa en las escuelas primarias públicas del Estado de Sonora, México. Se empleó una metodología cuantitativa de tipo descriptivo-correlacional; se aplicó el cuestionario-escala sobre la integración e inclusión de personas con necesidades educativas diversas a una muestra de 285 profesores de educación primaria del Estado de Sonora. Para el análisis de datos se utilizó el programa estadístico SPSS, versión 21 y el programa EQS versión 6.1, obteniendo datos de fiabilidad descriptivos, correlaciones y el modelo estructural. Los resultados indican que el modelo planteado en su conjunto predice en un $44 \%$ la inclusión educativa. Se puede concluir que las actitudes se relacionan recíprocamente con la cultura, afectando significativamente a la práctica docente, y son elementos claves en el proceso de inclusión educativa.

Palabras clave: actitudes; cultura; práctica docente; inclusión educativa; infancia intermedia.
Recibido: 27-septiembre-2017 / Aprobado: 21-marzo-2018

\begin{abstract}
The main objective of this labor is to analyze psychosocial aspects (attitudes, culture of diversity and teaching practice) to determine their relationship in the process of educational inclusion in public elementary schools at the State of Sonora, Mexico. A descriptive-correlational quantitative methodology was used; the questionnaire-scale on the integration and inclusion of people with diverse educational needs was applied to a sample of 285 elementary school teachers from the State of Sonora. For the data analysis, the statistical program SPSS, version 21 and the EQS program version 6.1 were used, obtaining descriptive reliability data, correlations and the structural model. The results indicate that the model proposed as a whole predicts educational inclusion by $44 \%$. We can conclude that attitudes are reciprocally related to culture, significantly affecting the teaching practice, being key elements in the process of educational inclusion.
\end{abstract}

Keywords: attitudes; culture; teaching practice; educational inclusion; intermediate childhood.

\footnotetext{
1 Licenciada en Psicología, maestra y doctora en Ciencias Sociales, Universidad de Sonora. Profesora del Departamento de Psicología y Ciencias de la Comunicación, Universidad de Sonora (México). Correo electrónico: reyna.campaal@gmail.com

2 Licenciado en Ciencias de la Comunicación, Universidad de Sonora. Máster en Periodismo Avanzado en Investigación, Universidad Ramón Llull. Maestro y doctor en Ciencias Sociales, Posgrado Integral en Ciencias Sociales (PICS) de la Universidad de Sonora. Profesor investigador de tiempo completo del Departamento de Psicología y Ciencias de la Comunicación, Universidad de Sonora (México). Miembro del Grupo de Enseñanza e Investigación de la Comunicación en América Latina (GEIC-AL). Correo electrónico: renecazarez@gmail.com
} 


\section{Introducción}

La Organización Mundial de la Salud (OMS, 2009) menciona que durante la infancia se experimenta un crecimiento rápido en el que influye la interacción que se establece con el entorno. En los primeros años de vida ocurren cambios en el sistema nervioso central, el cerebro triplica su tamaño y alcanza el $80 \%$ del peso adulto, de modo que las redes neuronales y las ventanas de aprendizaje que se abren y desarrollan durante este período determinarán la capacidad de aprendizaje de los niños y niñas. Por su parte, Bedregal y Pardo (2004) mencionan que este período crítico se transforma en el eje central del desarrollo integral y que en ningún momento posterior vuelve a ocurrir este fenómeno.

Este período representa un proceso complejo en el cual se sustenta la evolución biológica, psicológica y social en los primeros años de vida; se configuran las habilidades perceptivas, motrices, cognitivas, lingüísticas y sociales, las cuales posibilitarán una equilibrada interacción con el mundo

10 circundante (Fernández y Riquelme, 2006). Por su parte, Campo, Mercado, Sánchez y Vergara (2010) mencionan que para comprender el desarrollo existen dimensiones que permiten y hacen más claro su análisis, estableciendo cuatro áreas fundamentales: físico, perceptivo-cognitivo, afectivo-social y lenguaje.

Durante la infancia intermedia, que comprende entre los 6 a 12 años, los niños y niñas pueden concentrarse por más tiempo que los más pequeños y enfocarse en la información que necesitan. Es decir, hay un aumento en la atención selectiva, esto es debido a la maduración neurológica, que influye en el progreso de la memoria durante esta etapa del desarrollo (Papalia, Wendkos, Sally y Duskin, 2010). Por lo que la asistencia y la inclusión de los niños y niñas al nivel básico los hará poseedores de múltiples conocimientos y experiencias propias.

En el contexto educativo se configura una gran diversidad humana, siendo evidentes factores como el género, etnia, cultura, nivel socioeconómico, diferentes capacidades, entre otros (Guillén y Valenzuela, 2015). De igual manera, Barrero (2016) menciona que la población dominante en las escuelas son los estudiantes que se encuentran en riesgo o vulnerabilidad, es decir, la diversidad educativa. Por lo que educar en la diversidad desde los entornos inclusivos se fundamenta en los principios de equidad e igualdad, siendo un derecho que todos los seres humanos poseemos (Arnáiz, 2011).

Organismos internacionales como la Organización de las Naciones Unidas (ONU), el Fondo de las Naciones Unidas para la Infancia (Unicef) y la Organización de las Naciones Unidas para la Educación, la Ciencia y la Cultura (Unesco) han convocado una serie de acciones y reuniones internacionales a favor de que la educación llegue a todos los niños, sin distinción, en igualdad de condiciones y dentro del sistema educativo ordinario (Florian, 1998). Hacer efectivo el derecho a la educación exige garantizar que todos los niños, niñas y jóvenes tengan, en primer lugar, acceso a la educación, pero no a cualquier educación sino a una de calidad con igualdad de oportunidades (Bargerhuff, Cole y Teeters, 2012).

A pesar de la elaboración de políticas públicas en materia de educación y derechos humanos, aún impera la exclusión social en la infancia. Esta es entendida como la situación en que se encuentran los niños y niñas cuando no pueden participar plenamente en la vida social, económica y civil, o cuyo entorno personal, familiar, social y cultural no les permite acceder a un nivel adecuado de ingresos y recursos que supone que no puedan disfrutar de un nivel y una calidad de vida considerado aceptable por la sociedad en la que viven. Se puede decir que es cuando no se ejercen plenamente sus derechos fundamentales (Subirats, 2013).

Según la OMS (2011), se estima que hay más de 785 millones de personas con discapacidad en el mundo, lo que representan aproximadamente el $15,6 \%$ de la población mundial. Existen cerca de 95 millones de niños $(5,1 \%)$ con algún tipo de discapacidad, 13 millones de los cuales $(0,7 \%)$ tienen discapacidad grave. Se estima que el $80 \%$ de ellos viven en países de bajos ingresos con poco o ningún acceso a los servicios. De los aproximadamente 150 millones de niños con discapacidad en el mundo, solo el 2-3\% de los que viven en países de bajos ingresos van a la escuela (Banco Mundial, 2009). La Unesco (2014), en su undécimo informe de seguimiento de la educación para todos (EPT), indica que alrededor de 175 millones de niños y 
jóvenes son analfabetas y más de un tercio de esta población son discapacitados.

En México, 34 millones de personas están en rezago educativo, entre ellas 8,9 millones son analfabetas, 3.537 .926 de niños y jóvenes entre los 4 y 14 años de edad no asisten a la escuela y más de 1,3 millones tienen menos de 4 años de estudio (Inegi, 2016). El problema social equivale a un $13,42 \%$ de la población que debería estar gozando del derecho de educación básica. Mientras tanto, los datos de la Secretaría de Educación Pública (2014), para el ciclo 2013-2014 en el Estado de Sonora, el $86,8 \%$ de personas en edad entre 6 a 12 años realizan su estudio en educación primaria y el 13,8\% no acuden a la escuela.

Las cifras anteriores reflejan indicadores de exclusión educativa, dicha situación permite plantearnos las siguientes interrogantes: ¿qué aspectos psicosociales se relacionan en el proceso de inclusión educativa?, ¿las actitudes representan una barrera o favorece la inclusión?, ¿la cultura es un aspecto para la inclusión o exclusión?, ¿cuál es la formación y práctica docente en atención a la diversidad e inclusión?

Tal panorama representa un reto en el sistema educativo, en la promoción de políticas y prácticas de inclusión que permita a los estudiantes alcanzar los aprendizajes básicos de la educación obligatoria (Montiel y Arias, 2017). Debido a que durante la etapa escolar el niño empieza a desenvolverse, hacerse más autónomo de sus acciones, se forma el auto-concepto y la personalidad (Rice, 1997). Por su parte, Blanco (2012) menciona que la educación tiene varios beneficios en los primeros años de vida del individuo, ya que favorece el desarrollo, reduce tempranamente las desigualdades, tiene una influencia positiva en las trayectorias educativas y en el aprendizaje.

Es elemental, según Subirats (2013), que en el proceso de inclusión se reconstruya la condición de los actores sociales; siendo un proceso compartido en este caso por los profesores, padres de familia, estudiantes y sociedad en general en el que se busca que se reconozca la importancia de la educación y la significación de la acción educativa de todos. De igual forma, se puede considerar que la inclusión educativa constituye una forma de responder y abordar a la diversidad en el contexto educativo, con una ampliación y democratización de oportunidades de formación y en un ambiente de aprendizaje que promueva el desarrollo integral, personal y académico de todos los estudiantes (Lehohla y Hlalele, 2012).

En esta lógica, según Avellaneda (2015), se considera la importancia de establecer un posicionamiento crítico que permita visualizar, entender y realizar prácticas educativas a través de procesos y escenarios que propicien la socialización a partir del reconocimiento de las potencialidades humanas y como ciudadanos con el pleno derecho a la educación.

Con lo planteado anteriormente, el objetivo principal del presente estudio es analizar los aspectos psicosociales de actitudes, cultura y práctica docente para determinar su relación en el proceso de inclusión educativa en las escuelas primarias públicas del Estado de Sonora, México.

Dentro de este contexto, el presente artículo se estructura partiendo del marco teórico que permite definir los principales conceptos derivados del objeto de estudio, como los aspectos psicosociales: actitudes, cultura hacia la diversidad, práctica docente, diversidad e inclusión educativa. En segundo término, se describe la metodología de investigación, desde el enfoque y tipo de estudio, los participantes, técnicas de recolección de datos y el procedimiento empleado. En un tercer apartado se describen los resultados cuantitativos y, por último, se muestran las conclusiones y discusiones de la investigación.

\section{Marco teórico}

\section{Diversidad e inclusión educativa}

La sociedad actual se caracteriza por la creciente diversidad en cuanto a clases, género, cultura, características físicas o psicosociales. Así, se puede definir diversidad como las diferencias entre los individuos en cualquier atributo y es una característica individual del ser humano (Grütter y Meyer, 2014; Gómez, 2013). Estas diferencias pueden ser tanto físicas, psicológicas o sociales. En el Diccionario de la Real Academia Española (Drae, 2014) la palabra diversidad hace alusión a variedad, semejanza, diferencia; pero en una segunda acepción dice: abundancia, concurso de varias cosas distintas. 
Según González, Martín, Flores, Jenaro, Poy y Gómez (2013), la diversidad es un potencial enriquecedor para todos los individuos involucrados. En este mismo sentido, se expresa que el contexto educativo como sistema ofrece a los estudiantes: contenidos académicos adaptados a las necesidades educativas, interacciones colaborativas entre los miembros; para estimular, fomentar, ampliar las oportunidades de aprendizaje y erradicar barreras para su inclusión (Jiménez, Lalueza y FardeIla, 2017).

La recomendación internacional insiste en la necesidad de plantear la educación inclusiva como algo inacabado, que empieza en los primeros años de vida y se mantienen hasta que se acaba (Casanova, 2011, p. 95). Por lo cual, el papel que tienen las escuelas es ofrecer las herramientas acordes a sus características particulares, de manera que todos y cada uno de los estudiantes de la comunidad educativa se sientan acogidos, seguros y convencidos de que lograrán sus metas.

Por ello, la inclusión educativa se considera un proceso de cambio que permite abordar las necesidades de los estudiantes con alguna diversidad, pues tiene su base en los derechos humanos promoviendo la equidad y justicia social (Fasting, 2013; Mäkinen, 2013). En tal proceso es esencial la participación de los actores educativos en los centros escolares; lo que implica cambios en las adaptaciones curriculares, en materiales, metodología de trabajo y en la evaluación (Parrilla y Sierra, 2015).

En consecuencia, la educación tiene la función de garantizar la orientación adecuada, tanto del niño/niña y de la familia para lograr un sistema de influencia positivas necesarias que le permitan al infante un óptimo desarrollo psíquico y emocional estable (Ochoa y Nossa, 2013). En ese sentido, la Unesco (2017) menciona que las instituciones educativas deben proporcionar los espacios físicos adecuados, siendo una condición necesaria para generar ambientes propicios para el aprendizaje y el desarrollo de habilidades.

Aspectos psicosociales en el proceso de inclusión educativa

Durante el proceso de inclusión educativa es fundamental el ajuste y adaptación del niño, el cual dependerá de diversos aspectos psicosociales. Por ejemplo, las características individuales y las características del ambiente inmediato o distante en el que se desarrolle y que pueden aumentar la posibilidad de que manifiesten dificultades en el proceso de inclusión. Asimismo, son importantes en la interacción de variables del entorno, como, por ejemplo, la influencia de los pares, la cultura, la comunidad, la escuela y variables individuales como características cognitivas y temperamentales (Trudel y Puentes, 2000).

Un estudio realizado por McBrayer y Wong (2013) indica que la inclusión educativa requiere cambios significativos de valores, sistema y prácticas, siendo estos elementos esenciales en tal proceso. Centra una atención vital en el trabajo colaborativo entre profesores y contar con una visión compartida entre los actores educativos involucrados; además de la importancia de recursos materiales y la formación adecuada de los profesores. Tünnermann (2011) plantea que la interacción social produce un favorecimiento del aprendizaje mediante la socialización entre los alumnos, esto ayuda en la conformación de un ambiente de aprendizaje constructivista que facilita que todas las personas se conozcan para crear un clima con objetivos y metas comunes.

De Boer, Pij y Minnaert (2011) realizaron una revisión de 26 estudios sobre las actitudes de los profesores hacia la inclusión de los estudiantes con necesidades educativas especiales. En dicho estudio se evidenció que los profesores tienen actitudes favorables hacia la educación inclusiva. Las variables que se relacionan con la actitud son: la experiencia docente, la formación de los profesores y el tipo de diversidad; los efectos que se encontraron al tener actitudes favorables fue mayor participación social entre los alumnos en el aula.

En relación a lo anterior, los profesores tienen un papel clave en el desarrollo de las escuelas inclusivas. Diversos estudios realizados por Kgothule y Hay (2013), Forlin y Chambers, (2011), Malinen, Savolainen, Engelbrecht, Jiacheng, Nel, Nel y Tlale, (2013) evidencian la importancia que tienen las prácticas docentes en el fomento de la inclusión en el aula e indican que aquellos profesores que tienen una formación adecuada sobre la atención 
a la diversidad e inclusión educativa muestran mayor eficacia y satisfacción laboral; a diferencia de los que no cuentan con la formación adecuada, a quienes la presencia de la diversidad les produce estrés e insatisfacción laboral.

En virtud de las investigaciones anteriormente señaladas, se considera que los aspectos psicosociales que representan un papel primordial en el proceso de inclusión son las actitudes, la cultura inclusiva y la práctica docente.

\section{Actitudes hacia la diversidad}

En la Declaración de Salamanca de la Unesco sobre necesidades educativas: acceso y calidad, se afirma lo siguiente:

Los centros educativos ordinarios que ofrecen esta orientación inclusiva constituyen el medio más eficaz para combatir las actitudes discriminatorias, para crear comunidades de aceptación, para crear una sociedad inclusiva y para lograr una educación para todos, aún más: ofrecen una educación eficaz para la mayoría de los niños y mejoran la eficiencia y, en último extremo, el coste eficacia de todo el sistema educativo. (Unesco, 1994, p. IX)

En este sentido, Arnáiz expresa que:

Lo verdaderamente importante es que impregnen y cambien los pensamientos y actitudes y se traduzcan en nuevos planteamientos de solidaridad, de tolerancia y en nuevas prácticas educativas que traigan consigo una nueva forma de enfrentarse a la pluralidad y a la multiculturalidad del alumnado. (2005, p. 43)

Se construye así la capacidad que debe tener la escuela inclusiva de aceptar a todos los alumnos que deseen participar en ella y, de este modo, reducir la exclusión de los mismos.

Siendo las actitudes un aspecto relevante en el estudio de la inclusión, debido a que orienta a la ejecución de determinadas conductas, en tanto que son responsables de la tendencia que lleva implícita; a la vez incide en el desempeño profesional sobre las competencias (Aguilera, 2016). Por otra parte, en palabras de Rodríguez (2015), se afirma que el desconocimiento de la diversidad y de la inclusión causa en la sociedad determinados prejuicios, bajas expectativas y falsas creencias sobre las posibilidades de crecimiento en los alumnos, por lo que es necesario el empleo de estrategias formativas tanto en el profesorado, padres de familia y alumnos para la promoción y fomento de la inclusión.

\section{Cultura hacia la diversidad}

La cultura representa otro aspecto para el estudio de la inclusión, debido a que en un contexto educativo es fundamental conocer las significaciones que los miembros tienen hacia la diversidad y a las necesidades educativas especiales de los niños y niñas; a la vez, conocer las prácticas institucionales, creencias y valores; y si dicha cultura influye y de qué forma en la inclusión educativa de los estudiantes.

Según García (1997), se puede definir la cultura como el conjunto de los procesos sociales de producción, circulación y consumo de la significación en la vida social. Aplicando esta definición en las condiciones de multiculturalidad encontramos cómo un mismo objeto puede transformarse en uso social. El objeto se transforma y cambia de significado al pasar de un sistema cultural a otro; al insertarse en nuevas relaciones sociales y culturales.

Por su parte, Giménez (1995) indica que la cultura es dimensión simbólica-expresiva de todas las prácticas e instituciones sociales. Universo de informaciones, valores y creencias que dan sentido a nuestras acciones y al que recurrimos para entender el mundo. Este universo de sentido se expresa a través de un sistema de significantes que lo representan (símbolos de participación, solidaridad, jerarquía, evocación del pasado, símbolos nacionales, étnicos, religiosos, etc.).

López (2006) indica que la cultura de la diversidad es la lucha contra las desigualdades y segregación, se basa a partir de los principios éticos en los que creemos e implica un valor simbólico de estar constantemente en lucha contra la discriminación. Esta manera de pensar está basada en el sentido que le demos a la cultura de la diversidad como sinónimo de integración o de inclusión; esto 
implica, por lo tanto, una sociedad que modifique sus comportamientos y actitudes con respecto a los colectivos.

Por lo anterior, se puede considerar a la cultura como un aspecto psicosocial importante en el proceso de inclusión debido a que, dentro de una sociedad, se configura la socialización que es un fundamental para el desarrollo y vida como ciudadanos. Berger y Luckman (2006) refieren que la sociedad es un espacio mediador de afectividad interpersonal el cual es vivencialmente experimentado por el sujeto. En tal sentido, Vygotski (1984), haciendo alusión a la teoría sociocultural, propone dos zonas de desarrollo en las que se distingue el nivel real de desarrollo, determinado por la capacidad de resolver un problema, y el nivel de desarroIlo potencial, determinado a través de la resolución de un problema bajo la guía del adulto o en colaboración con otro compañero.

\section{Práctica docente}

14 Ante las exigencias actuales es necesario que el profesorado cuente con una formación propicia para construir su propio mapa de competencias profesionales con las que desarrolla su labor, en colaboración constante con el colectivo escolar con el que comparte la tarea docente para progresar en equipo y con alta implicación institucional (Domínguez, Leví, Medina y Ramos, 2014). El enfoque inclusivo busca promover en el profesorado una formación teórico-práctica, configurando actitudes favorables hacia la diversidad e inclusión; y con el conocimiento del desarrollo de las personas con necesidades educativas asociadas a la discapacidad (García, Massani y Bermúdez, 2016).

Medina, De la Herrán y Sánchez (2011) plantean que, bajo este contexto educativo, los profesores deben estar preparados para asumir, también, su rol de líderes sociales en la atención a la diversidad. Por lo tanto, es importante que, desde el nivel de educación básica hasta la educación superior, el profesorado cuente con una formación adecuada que dé respuesta a las necesidades de los estudiantes; así como contar con constantes capacitaciones que permitan dentro del aula resolver conflictos, propiciar una comunicación y motivación en los alumnos (Serrano y Pontes, 2017).
Raimilla y Morales (2014) indican que la práctica educativa en torno a la diversidad implica un proceso amplio y dinámico de construcción de conocimientos que surge a partir de la interacción e involucra valores, percepciones, capacidades y estilos de aprendizaje. En este, el docente emplea estrategias y procedimientos educativos diversificados y flexibles con la finalidad de dar respuesta a una realidad heterogénea y de contribuir a la mejora y al enriquecimiento de los estudiantes.

Por su parte, Gento y Sánchez (2010) plantean las siguientes características en la práctica educativa del profesorado en el contexto diverso de aprendizaje: puede ser de carácter interdisciplinario, se relaciona la teoría y la práctica y permite realizar innovaciones educativas. En relación a la diversidad, esta significa asumirla como una pedagogía del diálogo, reconociendo los diversos ritmos de aprendizaje, siendo la escuela un espacio democrático (Espinosa, 2017). Por lo que es importante el empleo de guías que organicen los objetivos, contenidos y metodologías para evaluar los aprendizajes adquiridos por los estudiantes.

\section{Metodología}

El enfoque de la presente investigación fue cuantitativo, el cual se utiliza para la recolección y el análisis de datos. Se utilizó la medición numérica, el conteo y la frecuencia en el uso de la estadística para establecer con exactitud patrones de comportamiento en una población (Hernández, Fernández y Baptista, 2010). Se empleó un estudio descriptivo-correlacional para poder establecer una correlación pues se midió la relación entre dos o más variables (Tamayo, 2003). Se utilizó un diseño no experimental-transeccional, ya que al ser no experimental las variables a trabajar no se ven manipuladas, basándose en la observación de fenómenos tal y como se dan en su contexto natural, es decir, los sujetos de estudio no se ven expuestos a estímulos; y al ser transeccional la investigación se centra en analizar el nivel o estado de una o diversas variables en un momento dado, o bien, conocer la relación entre un conjunto de variables en un punto temporal establecido (Taylor y Bodgan, 2004). La recolección de los datos se dio en un solo momento (2016-2017). 


\section{Participantes}

La muestra fue no probabilística por conveniencia, debido a que se tomó un subgrupo de la población y se emplearon una serie de criterios para la selección de los participantes. Los sujetos en total fueron 285 profesores de educación primaria, un 56\% son de sexo femenino y un $44 \%$ de sexo masculino. El nivel educativo del profesorado, un $67 \%$ cuenta con licenciatura, 20,8\% con maestría, $8,5 \%$, con especialidad y un $3,8 \%$ con diplomado; los años de antigüedad docente oscilan entre 1 a 40 años, con una media entre 11-15 años y una desviación estándar de 1,896.

Los criterios de selección de los participantes fueron los siguientes: contexto educación primaria del sector público, escuelas primarias con apoyo de los servicios y centros para la educación especial y la educación inclusiva en México y se consideraron todos los grados educativos de primero a sexto año.

\section{Instrumentos}

Se aplicó el cuestionario-escala sobre la integración e inclusión de personas con necesidades educativas y diversas (Gento, 2007). Este tiene como objetivo medir las condiciones educativas que se requieren en las escuelas primarias públicas en el Estado de Sonora para atender a la diversidad de alumnado e inclusión. Se retomaron las siguientes escalas: actitud de integración e inclusión, práctica docente, cultura inclusiva, diversidad e inclusión educativa. La escala de evaluación utilizada es de tipo likert, con opciones 1 (nunca), 2 (escasamente), 3 (frecuentemente), 4 (casi siempre) y 5 (siempre). La aplicación fue a lápiz y papel y con una duración de aplicación entre 25-30 minutos, aproximadamente. El coeficiente alfa de Cronbach del cuestionario fue ,83, lo que demuestra una consistencia interna adecuada.

\section{Procedimiento}

Para la aplicación de los instrumentos se acudió en primer lugar a los directivos para tener la autorización de realizar el estudio; se presentó una carta explicativa con los fines de la investigación. Una vez obtenida, se prosiguió a reunir a los participantes para tener su consentimiento para participar. Los profesores de las escuelas primarias seleccionadas fueron de los municipios de Hermosillo, Guaymas, Empalme y Ures, del Estado de Sonora.

\section{Análisis de datos}

Se empleó la base estadística de datos SPSS versión 21.00; se realizó un análisis estadístico descriptivo de las escalas del instrumento; se obtuvo la alfa para evaluar la confiablidad y consistencia interna; así como la media, mediana, moda, desviación estándar y correlaciones de Pearson. Estos métodos estadísticos son útiles para analizar dos o más variables independientes sobre una dependiente, la correlación nos da a conocer si existe relación significativa entre dos o más variables.

Se empleó el programa EQS versión 6.1 para realizar el modelo estructural. Esta es una técnica estadística multivariada para representar interrelaciones entre variables a partir de regresiones, en la que se analiza la influencia directa e indirecta de unas variables sobre otras (Bentler, 2006). Para determinar la pertinencia del modelo propuesto se utilizaron indicadores de bondad de ajuste.

Para medir la bondad de ajuste del modelo existen dos tipos de indicadores: el indicador estadístico chi cuadrado $\left(X^{2}\right)$ que determina la diferencia entre el modelo teórico propuesto y un modelo saturado formado por las relaciones entre todas las variables. Si el modelo teórico es pertinente este no es diferente del saturado, por lo que $X^{2}$ tendrá un valor alto y no significativo ( $p>.05)$; y los indicadores prácticos Bentler-Bonett de Ajuste Normado $(B B N F I)$ y Bentler- Bonettt de Ajuste No Normado $(B B N N F I)$.

\section{Resultados}

Para la validez del instrumento, se obtuvieron las alfas de Cronbach de las escalas que integran el instrumento; los resultados se muestran en la tabla 1. Como se puede observar, el alfa total fue de ,83, lo cual indica fiabilidad en el instrumento; a su vez, las alfas obtenidas en cada una de las escalas fueron mayor de ,60 por lo cual se consideran aceptables y fiables.

En la tabla 2 se muestran los resultados de la escala de actitudes. Se obtuvieron medias altas en el reactivo, por lo que los maestros muestran afinidad hacia la diversidad humana $(4,48)$ y en 
aceptación que todos los alumnos poseen conocimientos de los que hay que partir $(4,16)$. Sin embargo, se encontró una media baja de 2,90 que indica que escasamente los profesores consideran que los alumnos con diversidad pueden desarrollar sus propias potencialidades.
En la tabla 3 se muestran los resultados encontrados referentes al tipo de diversidad y grado; estos indican una media alta en el tipo de diversidad motora $(4,14)$ y socio-familiar $(4,41)$; por otro lado, las medias bajas fueron diversidad intelectual $(3,94)$ y visual $(3,98)$.

Tabla 1. Alfas de Cronbach de las escalas del instrumento.

\begin{tabular}{lllllll}
\hline Escalas & N & Mínimo & Máximo & Media & D.E & Alfa \\
& & & & &, 83 \\
Actitudes hacia la inclusión. & 285 & 1 & 5 & 4,34 &, 677 &, 74 \\
Cultura hacia la diversidad. & 285 & 1 & 5 & 4,22 &, 725 &, 73 \\
Práctica docente. & 285 & 1 & 5 & 4,31 &, 654 &, 89 \\
Diversidad. & 285 & 1 & 5 & 4,09 &, 673 &, 89 \\
Inclusión educativa. & 285 & 1 & 5 & 3,88 &, 783 &, 81 \\
\hline
\end{tabular}

Fuente: elaboración propia de los autores.

Tabla 2. Descriptivos de actitudes hacia la diversidad.

\begin{tabular}{lcccc}
\hline & Media & Mediana & Moda & $\begin{array}{c}\text { D.E. } \\
\text { Cronbach }\end{array}$ \\
\hline $\begin{array}{l}\text { Actitudes } \\
\begin{array}{l}\text { Aceptación que todos los alumnos poseen cono- } \\
\text { cimientos de los que hay que partir. }\end{array}\end{array}$ & $\mathbf{4 , 1 6}$ & 5,00 & 5 & 1,114 \\
$\begin{array}{l}\text { Fomenta la inclusión educativa en el aula. } \\
\text { Muestra afinidad hacia la diversidad humana. }\end{array}$ & 4,01 & 4,00 & 5 & 1,191 \\
$\begin{array}{l}\text { Los alumnos con diversidad pueden desarrollar sus } \\
\text { propias potencialidades. }\end{array}$ & $\mathbf{4 , 4 8}$ & 5,00 & 5 &, 796 \\
$\begin{array}{l}\text { Me siento comprometido hacia la inclusión educativa. } \\
\text { Men }\end{array}$ & 3,65 & 4,00 & 3 & 1,486 \\
\hline
\end{tabular}

Fuente: elaboración propia de los autores.

Tabla 3. Descriptivos de tipo de diversidad.

\begin{tabular}{|c|c|c|c|c|c|}
\hline & Media & Mediana & Moda & D.E. & $\begin{array}{c}\text { Alfa de } \\
\text { Cronbach }\end{array}$ \\
\hline Tipos de diversidades & & & & &, 89 \\
\hline Intelectual. & 3,94 & 4,00 & 5,00 & 783 & \\
\hline Visual. & 3,98 & 4,00 & 5,00 & ,874 & \\
\hline Auditiva. & 3,99 & 4,00 & 5,00 & 840 & \\
\hline Motora. & 4,14 & 4,33 & 5,00 & ,942 & \\
\hline Conductual. & 4,06 & 4,00 & 5,00 & ,854 & \\
\hline Socio-familiar. & 4,41 & 5,00 & 5,00 & ,807 & \\
\hline
\end{tabular}


En lo concerniente a la práctica docente, se encontró que los profesores emplean con mayor frecuencia las adaptaciones curriculares, diagnósticos, involucran a los padres de familia en las actividades del alumno y el trabajo colaborativo entre alumnos. Mientras que, lo que en menor frecuencia emplean y reciben es materiales didácticos adaptados, horarios flexibles y capacitaciones y actualizaciones. En la figura 1 se muestran estos resultados.

En la tabla 4 se muestran los descriptivos de la cultura de la diversidad, encontrándose medias altas en cada uno de los elementos que integran la escala.

Lo que respecta a las correlaciones de Pearson, siendo significativas tanto las actitudes con la práctica docente, los tipos de diversidad, cultura de la diversidad y la inclusión educativa, en la tabla 5 se muestran los resultados.

En la figura 2, se obtuvo que en el modelo estructural las actitudes hacia la diversidad e inclusión predice a la práctica docente con una covarianza de ,49, así como la cultura hacia la diversidad con una covarianza de ,52. También se puede detectar una relación bidireccional entre la cultura de la diversidad y las actitudes con una covarianza de ,58. Por otro lado, la práctica docente afecta significativamente a la inclusión educativa con una covarianza de ,63. Se puede encontrar validez convergente en los constructos que integran cada uno de los factores con pesos estructurales mayores de ,5, los cuales indican significancia.

Los indicadores de bondad de ajuste fueron considerados aceptables, ya que se obtuvieron los siguientes valores: índice de ajuste normado de Bentler-Bonett $(\mathrm{BBNFI})=, 96$; índice de ajuste no normado de Bentler-Bonett (BBNNFI)= 97 y el índice comparativo de ajuste de Bentler $(\mathrm{CFI})=, 98$. Estos valores alcanzan el nivel mínimo aceptable de los indicadores prácticos para considerar que un modelo con buena bondad de ajuste es de ,90. El valor del chi cuadrado fue $X^{2}$ de $=67,87$ (9 gl). $p=$ 0,000 . La probabilidad no fue significativa, lo que indica que no existen diferencias entre el modelo teórico y el modelo saturado por las relaciones entre variables.

Por último, se obtuvo un valor de ,064 en RMSEA (residuo de cuadrados mínimos), lo cual está dentro de los parámetros considerados aceptables ,08. La $R^{2}$ fue ,44, esto significa que el modelo en su conjunto explica el $44 \%$ de la varianza efectos de la inclusión educativa en los alumnos con necesidades educativas especiales.

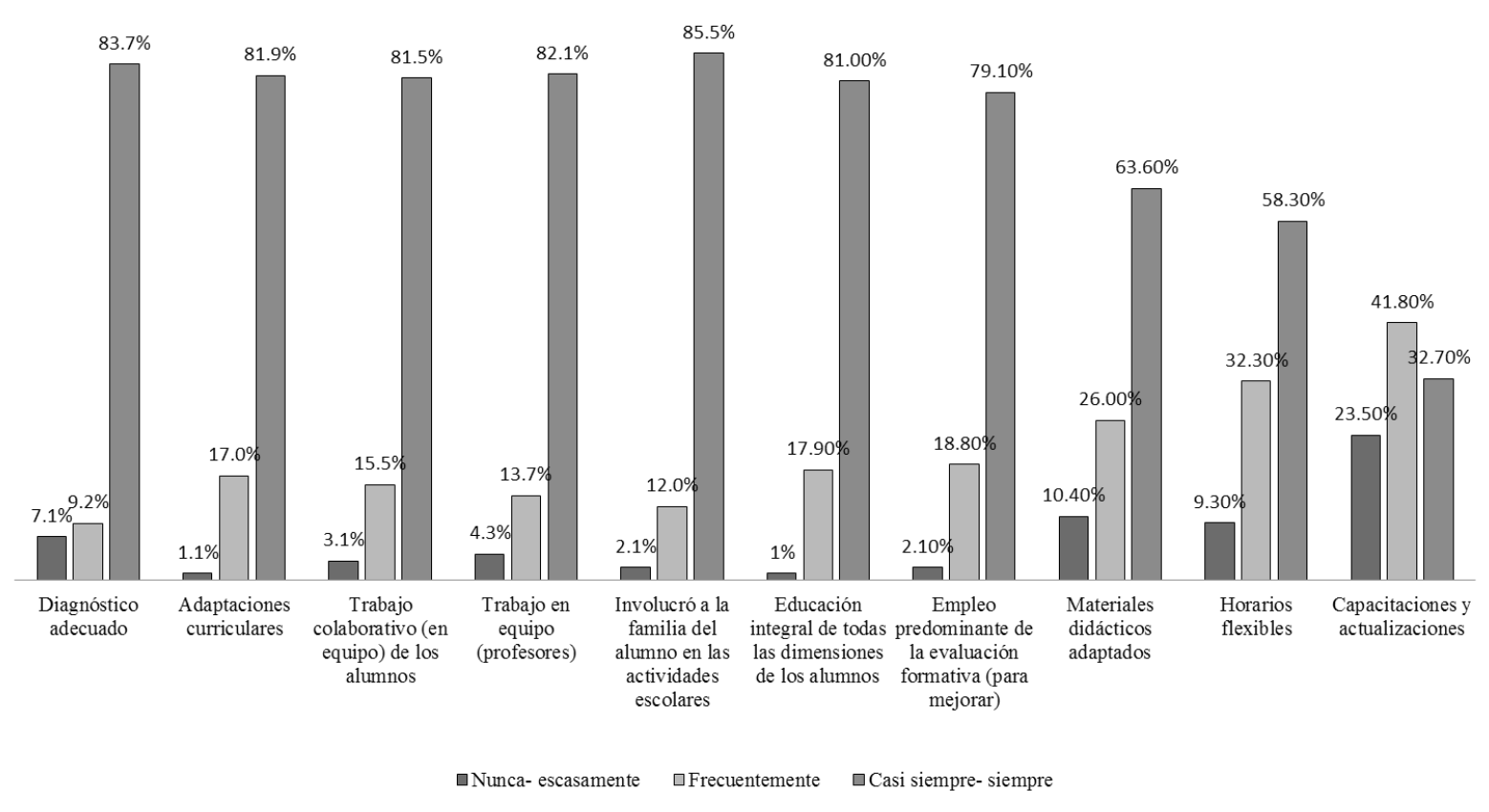

Figura 1. Práctica docente.

Fuente: elaboración propia de los autores. 
Tabla 4. Descriptivos de la cultura de diversidad en el aula.

\begin{tabular}{|c|c|c|c|c|c|}
\hline & Media & Mediana & Moda & D.E. & $\begin{array}{l}\text { Alfa de } \\
\text { Cronbach }\end{array}$ \\
\hline Cultura de diversidad & & & & & ,70 \\
\hline En el aula todos los alumnos se le hace sentir acogidos. & 4,60 & 5,00 & 5 & ,804 & \\
\hline Promuevo valores en el aula. & 4,92 & 5,00 & 5 &, 265 & \\
\hline Empleo la democracia en el aula. & 4,81 & 5,00 & 5 & ,393 & \\
\hline En la escuela se cuenta con una filosofía inclusiva. & 4,44 & 5,00 & 5 &, 757 & \\
\hline $\begin{array}{l}\text { Informo y promuevo la inclusión educativa (padres de } \\
\text { familia- alumnos). }\end{array}$ & 4,57 & 5,00 & 5 & ,717 & \\
\hline Hago vale el derecho de la educación. & 4,81 & 5,00 & 5 & ,435 & \\
\hline
\end{tabular}

Fuente: elaboración propia de los autores.

Tabla 5. Correlaciones entre las variables de estudio

\begin{tabular}{lccccc}
\hline & Actitudes & $\begin{array}{l}\text { Práctica } \\
\text { docente }\end{array}$ & $\begin{array}{l}\text { Tipos de } \\
\text { diversidades }\end{array}$ & $\begin{array}{l}\text { Cultura de la } \\
\text { diversidad }\end{array}$ & $\begin{array}{l}\text { Inclusión } \\
\text { educativa }\end{array}$ \\
\hline Actitudes. & 1 & - & - & - & - \\
Práctica docente. &, $644^{*}$ & 1 & - & - & - \\
Tipos de diversidades. &, $500^{* *}$ &, $486^{*}$ & 1 & - & - \\
Cultura de la diversidad. &, $600^{* *}$ &, $556^{* *}$ &, $510^{* *}$ & 1 & - \\
Inclusión educativa. &, $521^{* *}$ &, $689^{* *}$ &, $487^{* *}$ &, $621^{*}$ & 1 \\
\hline
\end{tabular}

** La correlación es significativa al nivel 0,01 (bilateral).

*La correlación es significante al nivel 0,05 (bilateral).

Fuente: elaboración propia de los autores.

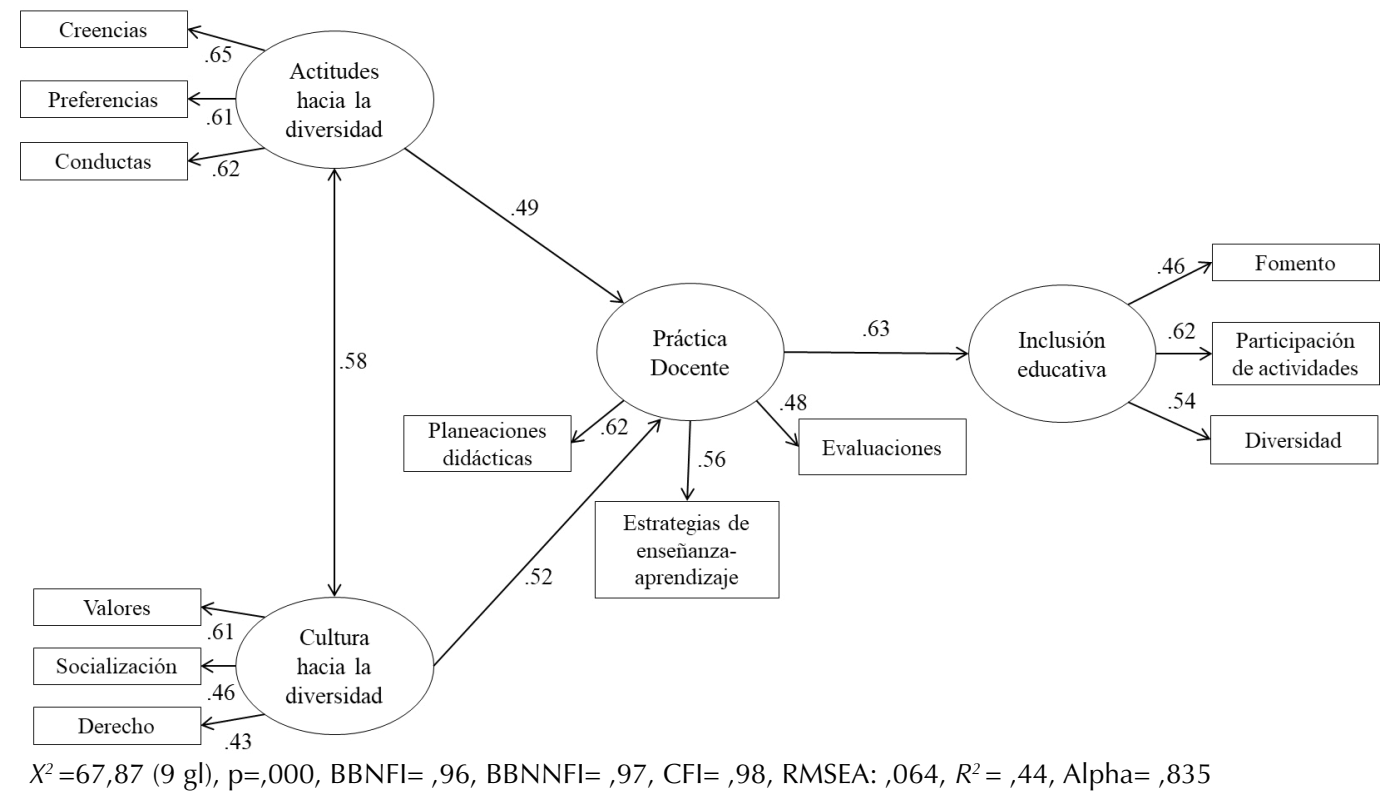

Figura 2. Modelo estructural de las variables del estudio.

Fuente: elaboración propia de los autores. 


\section{Conclusiones y discusiones}

La educación actual enfrenta un gran reto, tanto en el acceso a la educación como en su aprovechamiento. Tiene ante sí el desafío de preparar y desarrollar en los estudiantes las destrezas, habilidades y conocimientos suficientes para enfrentar los desafíos del mundo actual (Valenzuela, Guillén y Campa, 2014). En lo referente a la inclusión educativa, Casanova (2015) menciona que este nuevo modelo educativo supone un gran avance en la atención del alumnado con necesidades educativas especiales, principalmente en etapas básicas del desarrollo. Esto implica en las escuelas una restructuración en el currículum, ambientes de aprendizaje, actitudes, cultura y actividad pedagógica.

De acuerdo a los objetivos de investigación planteados, se puede señalar aquellos aspectos psicosociales asociados a la inclusión educativa. En lo correspondiente a la variable de actitudes se observa la presencia de creencias como la aceptación de los alumnos con diversidad, debido a que los profesores consideran que poseen conocimientos de los que hay que partir; a la vez, muestran afinidad hacia la diversidad humana. Sin embargo, muestran preferencias hacia las diversidades de tipo motora, socio-familiar y conductual, en grado leve y medio; y poca aceptación a diversidad intelectual, auditiva y visual en sus diversos grados de afectación. Por tanto, se evidencian actitudes poco favorables hacia la inclusión y esta se limita solamente para ciertos tipos de diversidades como ya se ha señalado.

Los autores Fishbein y Ajzen (1980), en su modelo de la teoría de acción razonada, indican que una actitud va a predecir determinadas conductas, las cuales se manifiestan en este caso en la práctica docente. En el estudio se encontró que los profesores realizan en sus haceres cotidianos en el aula adaptaciones curriculares y evaluaciones adaptadas a las necesidades de los niños y niñas. De igual forma, se cuentan con diagnósticos para determinar el grado de dificultad de los estudiantes. En relación a la práctica docente, se emplea el trabajo colaborativo como estrategia de enseñanza- aprendizaje. Es importante plantear un modelo pedagógico que guíe y organice la práctica docente, así como menciona De Zubiría (2008), plantear objetivos, contenidos, metodologías y detectar el desarrollo del infante.

Por su parte, Fernández (2013) refiere que las competencias requeridas en la función docente para atender a la diversidad han de caracterizarse en identificar las necesidades de los estudiantes, desarrollar estrategias innovadoras para la inclusión, realizar adaptaciones curriculares y conformar redes de apoyo escolar. De esta forma, es indispensable una constante capacitación profesional en: actualización y conocimiento adecuado de los tipos de diversidades, estrategias de enseñanza-aprendizaje, actividades de inclusión en el grupo y actividades específicas para la diversidad de los alumnos y adaptaciones curriculares. Elementos que resultan relevantes evaluar en próximas investigaciones para la mejora de la práctica docente.

Leithwood (2009) plantea un modelo de mejora de la escuela en el que la planificación debe realizarse de modo colaborativo, comprendiendo la realidad y las perspectivas de todos los agentes implicados, pues para lograr la inclusión educativa es necesario generar culturas inclusivas con respeto a la diversidad, implicando a la comunidad estudiantil y a la sociedad en general. En ese sentido, para Booth y Ainscow (2002), la conformación de culturas inclusivas implica crear una comunidad segura, abierta, colaboradora y estimulante en la que todos sean valorados, desarrollar valores inclusivos compartidos por todo el personal, los estudiantes, los directores y los padres y que son transmitidos a todos los nuevos miembros de la escuela.

El aspecto cultural es otro rubro que requiere atención. López (2006) refiere que el reconocimiento de la diversidad implica un modelo educativo que sea capaz de educar a una ciudadanía que comprenda, defienda y promueva las diferencias humanas como valor y derecho humano. Es decir, la cultura de la diversidad se basa en principios éticos y exige a la sociedad cambios en sus comportamientos con respecto a las personas segregadas. Se evidencia con los resultados encontrados en la investigación que los participantes promueven valores, emplean la democracia en el aula, socialización y se hace valer el derecho a la educación; esto promueve en las escuelas una cultura inclusiva hacia la diversidad. 
Urrego, Restrepo, Pinzón, Acosta, Díaz y BoniIla (2014) aluden que un desarrollo óptimo y sano depende en gran medida de las interacciones que se forjan en las etapas tempranas del ciclo vital; de igual forma, de las relaciones que establecen en el contexto social que se desenvuelve. Como advierten Contreras y Campa (2017), cuando se habla de socialización se trata en términos generales, como el proceso en el cual los individuos incorporan normas, roles, valores, actitudes y creencias a partir del contexto socio-histórico en el que se encuentran insertos a través de diversos agentes de socialización tales como familia, los grupos de pares y las instituciones educativas, entre otras. Por su parte, Jadue (2003), Oliva (2006), Valenzuela, Campa y López (2012) indican que los cambios que transcurre el ser humano durante la infancia tienen un impacto en el desarrollo psicosocial y los actores sociales, afectando significativamente la calidad de vida, tanto en el ámbito educativo como en las relaciones sociales que establece el niño o niña durante la etapa escolar.

Lo señalado anteriormente, y partiendo de los resultados obtenidos, permite enunciar los principales aspectos psicosociales asociados al proceso de inclusión educativa. Entre ellos, se señala en el estudio una relación significativa entre las actitudes y la práctica docente, así como la presencia de una cultura de diversidad, enmarcado en la igualdad de derecho y la promoción de valores. De igual forma, se emplean estrategias como el trabajo colaborativo con alumnos, fungiendo como ejes medulares en el proceso de inclusión. Se puede concluir que el modelo en su conjunto predice un $44 \%$ la inclusión educativa.

De tal manera, un aspecto relevante para próximas investigaciones es analizar la formación del profesorado en torno a la diversidad; siendo la práctica docente un pilar fundamental en los haceres cotidianos que tiene el profesor. Pues, tal como menciona Esteve (2010), para conseguir una educación de calidad es necesario el desarrollo de una formación de profesores adecuada para que los docentes puedan atender con éxito a estos niños y niñas con dificultades. En ese sentido, la Unesco (2015) plantea la construcción de políticas educativas que orienten las prácticas educativas. Por último, la Secretaría de Educación Pública (2014), siguiendo los lineamientos de la reforma educativa, recomienda la reorganización de los centros escolares, recursos, procesos y acciones a seguir para promover e impulsar la educación inclusiva.

\section{Referencias}

Aguilera, J. (2016). Actitudes hacia la discapacidad de aspirantes a educadores sociales. Propuestas de formación para una educación inclusiva. Revista Nacional e Internacional de Educación Inclusiva, 9(1), 13-29. Recuperado de http:// www.revistaeducacioninclusiva.es/index.php/ REl/article/view/67/64

Arnáiz, P. (2005). Fundamentos de la educación inclusiva. En C. Alba Pastor, M. P. Sánchez Hípola y J. A. Rodríguez (coords.), Jornadas de Cooperación Educativa con Iberoamérica sobre Educación Especial e Inclusión Educativa (pp. 25-43). Madrid: Ministerio de Educación, Universidad Complutense.

Arnáiz, P. (2011). Luchando contra la exclusión: buenas práctica y éxito escolar. Innovación Educativa, 21, 23-25. Recuperado de www.usc.es/ revistas/index.php/ie/article/download/22/138

Avellaneda, Y. T. (2015). Prácticas pedagógicas de formación política en la infancia. Infancias Imágenes, 14(1), 99-111. https://doi.org/10.14483/ udistrital.jour.infimg.2015.1.a07

Banco Mundial (2009). Informe sobre el desarroIlo mundial: una nueva geografía económica. Recuperado de http://documentos.bancomundial.org/curated/es/785111468331213672/ f/437380WDR20091101OFFICIALOUSE0ONLY1.pdf

Bargerhuff, M., Cole, D. y Teeters, L. (2012). Using a transdisciplinary model to address inequities in field placements for teacher candidates with disabilities. International Journal of Inclusive Education, 16(2), 185-206. https://doi. org/10.1080/13603111003721098

Barrero, M. (2016). Lo visible e invisible de la diversidad en la educación infantil. Infancias Imágenes, 15(2), 262-270. https://doi.org/10.14483/ udistrital.jour.infimg.2016.2.a07

Bedregal, P. y Pardo, M. (2004). Desarrollo infantil temprano y derechos del niño. Chile: Unicef. 
Recuperado de http://www.bvsde.paho.org/texcom/cd051479/bedregal.pdf

Bentler, P. 2006). EQS 6 Structural Equations Program Manual. Encino, CA: Multivariate Software, Inc.

Berger, L. y Luckman (2006). La construcción social de la realidad. Buenos Aires: Amorrortu.

Blanco, R. (2012). Inclusión educativa en América Latina: caminos recorridos y por recorrer. En A. Marchesi, Blanco, R. y Hernández, L., Educación inclusiva: avances y desafíos de la educación inclusiva en Iberoamérica. Madrid: Organización de Estados Iberoamericanos para la Educación, la Ciencia y la Cultura (OEI).

Booth, T. y Ainscow, M. (2002). Index for inclusion: Developing learning and participation in schools. Bristol: Centre for Estudies on Inclusive Education. Recuperado de http://www.eenet. org.uk/resources/docs/Index \%20English\%20 for\%20Malta.pdf

Campo, L., Mercado, L., Sánchez, L. y Vergara, C. (2010). Importancia de la estimulación de las aptitudes básicas del aprendizaje desde la perspectiva del desarrollo infantil. Psicogente, 13 (24), 397-41. Recuperado de http://revistas. unisimon.edu.co/index.php/psicogente/article/ view/1801/1717

Casanova, M. (2011). Educación inclusiva: un modelo de futuro. Madrid: Wolters Kluwers.

Casanova, M. (2015). El modelo de evaluación en una ley de consenso. Revista de la Asociación de Inspectores de Educación de España, 1(23), 1-12. Recuperado de https://avances.adide.org/ index.php/ase/article/view/22/23

Contreras, R. y Campa, R. (2017). Caracterización del perfil de los estudiantes de secundaria en el acceso y uso de internet a partir de las TIC. Edutec, Revista Electrónica de Tecnología Educativa, 61, 8-21. https://doi.org/10.21556/ edutec.2018.61

De Boer, A., Pijl, S. y Minnaert, A. (2011). Regular primary schoolteacher's attitudes towards inclusive education: A review of the literatura. International Journal of Inclusive Education, 15(3), 331-353. https://doi. org/10.1080/13603110903030089
Diccionario Real Academia Española (Drae) (2014). Diversidad. Recuperado en http://dle.rae. es/?id=EObOPXH

Domínguez, C., Leví, G., Medina, A. y Ramos, E. (2014). Las competencias docentes: diagnóstico y actividades innovadoras para su desarrollo en un modelo de educación a distancia. Revista de Docencia Universitaria, 12(1), 239-267. https://doi.org/10.4995/ redu.2014.6431

Espinosa, D. (2017). Hacia una pedagogía de la diversidad: pensar en la escuela. Repique, Revista de Ciencias Sociales, 1, 145-157. Recuperado de http://www.utelvt.edu.ec/revista/index.php/ Repique/article/view/25/24

Esteve, J. (2010). La tercera revolución educativa. La educación en la sociedad del conocimiento. Barcelona: Paidós.

Fasting, R. (2013). Adapted Education: The Norwegian pathway to inclusive and efficient education. International Journal of Inclusive Education, 17(3), 263-276. https://doi.org/10.1 $\underline{080 / 13603116.2012 .676083}$

Fernández, J. M. (2013). Competencias docentes y educación inclusiva. Revista Electrónica de Investigación Educativa, 15(2), 82-99. Recuperado de http://redie.uabc.mx/vol15no2/contenido-fdzbatanero.html

Fernández, L. y Riquelme, P. (2006). Sistematización programa de estimulación temprana: sistematización y ejecución de un modelo de estimulación temprana en población infantil. Claves para la replicabilidad. Santiago: Gobierno de Chile. Recuperado de http://www.crececontigo.gob.cl/wp-content/uploads/2015/11/14Programa-de-Estimulacion-Temprana.pdf

Fishbein, M. y Ajzen, I. (1980). Formación del profesorado para la diversidad cultural. Madrid: La Muralla.

Florian, L. (1998). Prácticas inclusivas. ¿Qué prácticas son inclusivas, por qué y cómo? En C. Tilstone, L. Florian y R. Rose (coords.), Promoción y desarrollo de prácticas educativas inclusivas. Madrid: EOS.

Forlin, C. y Chambers, D. (2011). Teacher preparation for inclusive education: increasing knowledge but raising concerns. Asia-Pacific 
of Teacher Education, 39(1), 17-32. https://doi. org/10.1080/1359866X.2010.540850

García, C. N. (1997). Cultura y comunicación: entre global y local. Buenos Aires: Universidad de la Plata. https://doi.org/10.1174/113839597322011951

García, X., Massani, E. y Bermúdez, I. (2016). La educación inclusiva en la formación de profesionales de la educación. Universidad y Sociedad, 8(1), 118-121. Recuperado de http:// scielo.sld.cu/pdf/rus/v8n1/rus17116.pdf

Gento, S. (2007). Requisitos para una inclusión de calidad en el tratamiento educativo de la diversidad. Bordón, 59(4), 581-595. Recuperado de: https://dialnet.unirioja.es/descarga/articulo/2582774.pdf

Gento, S. y Sánchez, C. (2010). El practicum en el tratamiento educativo de la diversidad. Madrid: Uned.

Giménez, G. (1995). Modernización, cultura e identidad social. Espiral, Estudios sobre Estado y Sociedad, 1(2), 35-56. Recuperado de: http://148.202.18.157/sitios/publicacionesite/ pperiod/espiral/espiralpdf/Espiral2/35-56.pdf

Gómez, I. (2013). Dirección y gestión de la diversidad en la escuela: hacia un liderazgo inclusivo. Fuentes, 14(1), 61-84. Recuperado de https://revistascientificas.us.es/index.php/fuentes/article/ view/2353/2172

González, F., Martín, E., Flores, N., Jenaro, C., Poy, R. y Gómez, M. (2013). Teaching, learning and inclusive education: The challenge of teachers' training for inclusion. Procedia Social and Behavioral Sciences, 93, 783-788. Recuperado de https://www.sciencedirect.com/science/article/pii/S187704281303382X https://doi.org/10.1016/j.sbspro.2013.09.279

Grütter, J. y Meyer, B. (2014). Intergroup friendship and children's intentions for social exclusion in integrative classrooms: The moderating role of teacher's diversity beliefs. Journal of Applied Social Psychology, 44, 481-494. https://doi. org/10.1111/jasp. 12240

Guillén, M. y Valenzuela, B. (2015). Capítulo 2. Diversidad e inclusión educativa en Sonora, México. Reto en construcción. En B. Valenzuela, M. Guillén y A. Medina, Procesos educativos: desafíos y retos en siglo XXI. México: Pearson.
Hernández, R., Fernández, C. y Baptista, P. (2010). Metodología de la investigación. Lima: McGraw-Hill. Instituto Nacional de Estadística y Geografía (Inegi) (2016). Encuesta nacional de ingresos y gastos de los hogares (ENIGH). Recuperado de http:// www.beta.inegi.org.mx/proyectos/enchogares/ regulares/enigh/nc/2016/

Jadue, G. (2003). Transformaciones familiares en Chile: riesgo creciente para el desarrollo emocional, psicosocial y la educación de los hijos. Estudios Pedagógicos, 29, 115-126. https://doi. org/10.4067/S0718-07052003000100008

Jiménez, F., Lalueza, J. y Fardella, C. (2017). Aprendizajes, inclusión y justicia social en entornos educativos multiculturales. Revista Electrónica de Investigación Educativa, 19(3), 10-23. https://doi.org/10.24320/redie.2017.19.3.830

Kgothule, R. y Hay, M. (2013). Educator's views on management practices in the implementation of inclusive education: An ecosystemic approach. Journal Human Ecologic, 42(1), 33-41. https:// doi.org/10.1080/09709274.2013.11906579

Lehohla, M. y Hlalele, D. (2012). Inclusive classrooms: An ecosystemic perspective. Journal of Human Ecology, 37(3), 189-201. Recuperado de https://www.researchgate.net/profile/ Dipane Hlalele2/publication/265741248 Inclusive Classrooms An Ecosystemic Perspective/links/5927e2f40f7e9b99799f17eb/ Inclusive-Classrooms-An-Ecosystemic-Perspective.pdf https://doi.org/10.1080/09709274.201 2.11906464

Leithwood, K. (2009). ¿Cómo liderar nuestras escuelas? Aportes desde la investigación. Santiago: Área de Educación, Fundación Chile. Recuperado de http://ww2.educarchile.cl/UserFiles/P0038/File/libros/Libro Liethwood.pdf

López, M. (2006). La ética y la cultura de la diversidad en la escuela inclusiva. Sinéctica, 29. Recuperado de http://rodin.uca.es/xmlui/ bitstream/handle/10498/7765/33194385. pdf? sequence $=1$

Mäkinen, M. (2013). Becoming engaged in inclusive practices: Narrative reflections on teaching as descriptors of teachers' work engagement. Teaching and Teacher Education, 35 51-61. https://doi.org/10.1016/j.tate.2013.05.005 
Malinen, O., Savolainen H., Engelbrecht, P., Jiacheng X., Nel, M., Nel, N. y Tlale, D. (2013). Exploring teacher self-efficacy for inclusive practices in three diverse countries. Teaching and Teacher Education, 33, 34-44. https://doi. org/10.1016/j.tate.2013.02.004

McBrayer, K. y Wong, P. (2013). Inclusive education services for children and youth with disabilities: Values, roles and challenges of school leaders. Children and Youth Services Review, 35, 1520-1525. https://doi.org/10.1016/j. childyouth.2013.06.009

Medina, A., De la Herrán, A. y Sánchez, C. (2011). Formación pedagógica y práctica del profesorado. Madrid: Universitaria Ramón Areces.

Montiel, G. y Arias, L. (2017). Práctica docente en atención de los alumnos con necesidades educativas especiales. Revista Electrónica sobre Tecnología, Educación y Sociedad, 4(7), Recuperado de www.ctes.org.mx/index.php/ctes/ article/download/653/733

Ochoa, S. y Nossa, L. (2013). La infancia desde las apuestas formativas en educación y política pública. Infancias Imágenes, 12(1), 18-27. https:// doi.org/10.6018/reifop.18.1.214381

Oliva, A. (2006). Relaciones familiares y desarrollo adolescente. Anuario de Psicología, 36(3), 209-223. Recuperado en http://www. raco.cat/index.php/AnuarioPsicologia/article/ view/61838/82584

OMS (2009). Desarrollo en la primera infancia. Recuperado en http://www.who.int/mediacentre/ factsheets/fs332/es/index.html

OMS (2011). Informe mundial sobre la discapacidad. Recuperado de http://www.who.int/disabilities/ world report/2011/accessible es.pdf

Papalia, D., Wendkos, O., Sally y Duskin, F. (2010). Desarrollo físico cognoscitivo en la infancia media. En, Desarrollo humano (pp. 292-317). $11^{\mathrm{a}}$ ed. México: McGraw-Hill.

Parrilla, A. y Sierra, S. (2015). Construyendo una investigación inclusiva en torno a las distintas transiciones educativas. Revista Electrónica Interuniversitaria de Formación del Profesorado, 18(1), 161-175. https://doi.org/10.6018/ reifop.18.1.214381
Raimilla, M. y Morales, S. (2014). Niños con necesidades educativas especiales. Requerimientos de familias frente al rol del educador inicial. Infancias Imágenes, 13(1), 8-22. https://doi. org/10.14483/issn.1657-9089

Rice, F. (1997). Desarrollo humano. Estudio del ciclo vital. México. Prentice.

Rodríguez, M. (2015). Actitudes hacia la discapacidad en alumnos de Magisterio de Educación Infantil. Propuestas de formación para una Educación Inclusiva. Revista Nacional e Internacional de Educación Inclusiva, 8(3), 137-152. Recuperado de http://www.revistaeducacioninclusiva.es/index.php/REl/ article/view/95/92

Secretaria de Educación Pública (SEP) (2014). Sonora educado. Informe 2013-2014. Recuperado de http://informe.sonora.gob. mx/wpcontent/uploads/2014/09/INFORME-2014-LIBRO-eje3-EDUCADO.pdf

Serrano, R. y Pontes. A. (2017). Diferencias entre expectativas y logros en las competencias del prácticum del Máster de Formación del Profesorado de Enseñanza Secundaria. Revista Electrónica Interuniversitaria de Formación del Profesorado, 20(1), 1-18. https://doi. org/10.6018/reifop/20.1.235151

Subirats, J. (2013). Una visión desde las políticas públicas en pleno cambio de época y con riesgos significativos de exclusión social. ATPS. Revista Internacional Animación, Territorios y Prácticas Socioculturales, 5, 13-26. Recuperado de http://www.atps.uqam.ca/numero/n5/ pdf/ATPS Subirats 2013.pdf

Tamayo, M. (2003). El proceso de investigación científica. México: Limusa Noriega.

Taylor, S. J. y Bogdan, R. (2004). Introducción a los métodos cualitativos de investigación. Barcelona: Paidós.

Trudel, M. y Puentes, G. (2000): The Contemporary Concepts of at Risk Children: Theoretical Models and Approaches in the Early Years. Recuperado de https://www.academia. edu/21479520/The Contemporary Concepts of At-Risk Children Theoretical Models and Preventive Approaches in the Early Years?auto=download 
Tünnermann, C. (2011). El constructivismo y el aprendizaje de los estudiantes. Universidades, 61(48), 21-32. Recuperado de http://www.redalyc.org/pdf/373/37319199005.pdf

Unesco (1994). Declaración de Salamanca y marco de acción para las necesidades educativas especiales. París: Unesco. Recuperado de http:// www.unesco.org/education/pdf/SALAMA S. PDF

Unesco (2014). Servicio en prensa niños sin escolarización. Recuperado de http://www.unesco. org/new/es/media-services/singleview/news/ global learning crisis is costing 129 billion a year/\#.VTKylfmG93Q

Unesco (2015). Educación inclusiva. Foro mundial sobre la educación 2015. Recuperado de http://unesdoc.unesco.org/images/0023/002332/233245s.pdf

Unesco (2017). Suficiencia, equidad y efectividad de la infraestructura escolar en América Latina según el Terce. Santiago de Chile: Unesco.
Urrego, Y., Restrepo, J., Pinzón, S., Acosta, J., Díaz, M. y Bonilla, C. (2014). Vínculo afectivo en pares y cognición social en la infancia intermedia. International Journal of Psychological Research, 7(2), 51-63. https://doi. org/10.21500/20112084.658

Valenzuela, B. A., Guillén, M., Campa, R. (2014). Recursos para la inclusión educativa en el contexto de educación primaria. Infancias Imágenes, 13(2), 64-75. DOI: https://doi. org/10.14483/udistrital.jour.infimg.2014.2.a06

Valenzuela, B., Campa, R. y López, M. (2012). Factores psicosociales asociados al imaginario social a partir de las percepciones de niños y jóvenes en El fuerte, Sinaloa. Revista de Arquitectura, Urbanismo y Ciencias Sociales Topofilia. Centro de Estudios de América del Norte y El Colegio de Sonora, 3(2). Recuperado de http://148.228.173.140/topofiliaNew/assets/ tres2nueve.pdf https://doi.org/10.14483/udistrital.jour.infimg.2014.2.a06

Vygotski, L. (1984). Aprendizaje y desarrollo intelectual en la edad escolar. Infancia y Aprendizaje, vol. 27/28, 105-116. Recuperado de https:// dialnet.unirioja.es/descarga/articulo/668448. pdf https://doi.org/10.1080/02103702.1984.1 $\underline{0822045}$ 